\title{
Formulation and Evaluation of Gastro-Bilayer Floating Tablets of Losartan Potassium as Immediate Release Layer and Ramipril Hydrochloride as Sustained Release Floating Layer
}

\author{
Syed Iftequar Ahmed ${ }^{1 *}$, Zahid Zaheer ${ }^{2}$, Furquan Nazimuddin Khan ${ }^{2}$, Moizul Hasan ${ }^{1}$ \\ 'Department of Pharmaceutics, Y.B. Chavan College of Pharmacy, Aurangabad, Maharashtra, INDIA. \\ ${ }^{2}$ Department of Quality Assurance, Y.B. Chavan College of Pharmacy, Aurangabad, Maharashtra, INDIA.
}

\begin{abstract}
Objectives: A sustained release Gastro-bilayer floating tablets with reduced dosing frequency and increased drug bioavailability is developed. The dosage form is suitable for the release of two drugs simultaneously in a single dosage unit i.e. Losartan Potassium and Ramipril Hydrochloride. The SeDeM system for selection of powder blend for direct compression gave the parameter index value of $\geq 0.5$ which is considered as best in terms of compression properties. The prepared dosage form provided sustained effect of the drug for $12 \mathrm{hr}$ by Non-Fickian, controlled diffusion and swollen matrix. Peppa's release model is best suited for the final formulation of batches. Optimized formulation exhibited floating time of $12 \mathrm{hr}$ and floating lag time of less than $1 \mathrm{~min}$. In-vitro dissolution studies shows $96 \%$ of the both the drugs release. The swelling and erosion of polymers is studied by Scanning Electron Microscopy. The data obtained from Analysis of variance demonstrated the significance of the model with a $P$-value of less than 0.05. Methods: SeDeM system was adopted on powder blend before direct compression for preparation of tablet, the hydroxyl propyl methyl cellulose was used as a rate-controlling polymer and a mixture of citric acid and Sodium bicarbonate formed the floating layer. Results: Radius values through SeDeM diagram was plotted and index values are calculated and resulted in an index value of $\geq 0.5$. The pre-compression and post compressional parameters are as per Indian Pharmacopeia specifications.
\end{abstract}

The best formulation for gastric bilayer tablet had a combination of Hydroxy propyl methyl cellulose K15M and Sodium Carboxy methyl cellulose which gave $99.57 \%$ drug dissolution within $12 \mathrm{hr}$. Conclusion: Gastro-Bilayer Floating Tablets of Losartan as Immediate Release layer and Ramipril as sustained-release floating layer are successfully developed. The problem of poor solubility of drugs can be solved by optimization of blend through SeDeM expert System. Hydroxy propyl methyl cellulose K15M, K4M and Carbopol 934 are helpful in achieving the sustained effect of the drug for 12 hr by Non-Fickian, controlled diffusion and swollen matrix. Peppa's release model is best suited for the final formulation batches based on the drug release mechanisms.

Key words: Gastro-Bilayer Floating Tablets, Losartan Potassium, Ramipril Hydrochloride, SeDeM, Sustained Release Floating Layer.

\section{Correspondence}

Mr. Syed Iftequar Ahmed

Department of Pharmaceutics, Y.B. Chavan College of Pharmacy, Aurangabad, Maharashtra, INDIA.

Phone no: +919028223979

Email: iftequar2212@gmail.com

DOI: 10.5330/ijpi.2020.3.53

\section{INTRODUCTION}

The aim of the study is to formulate controlled release Gastro-bilayer floating tablets that will reduce the dosing frequency and increase drug bioavailability for treatment of chronic hypertension. ${ }^{1}$ The dosage form is suitable for release of two different types of drugs, wherein one layer is of immediate release which releases Losartan Potassium and the second sustained release layer is of Ramipril Hydrochloride in a single dosage unit. This type of dosage form has many advantages over the conventional single-layered Tablets. Losartan potassium is an angiotensin II receptor antagonist and Ramipril Hydrochloride is angiotensin-converting enzyme-inhibitor when these two drug are used in combination blocks total production of angiotensin mostly responsible for hypertension. ${ }^{2}$ The advantages of such bilayer dosage form is mentioned in most of the literature and further up till now this type of combination formulation is still not available therefore such type of dosage form is developed. ${ }^{3}$ Formulating bilayer dosage form may contribute to cost-effectiveness by eliminating different coatings required to get sustained-release action. ${ }^{4}$ SeDeM expert system is a predictive tool that can be applied for evaluation of suitability, critical quality attributes and the behavior of the drug material and excipients used for the formulation development of bilayer tablets by direct compression having an impact on the final product. ${ }^{5}$ This system provides a physical profile of powder material intended to be used and suggests their flow performance. SeDeM expert system technique may reduce the number of preformulation trials and may be coined as time-saving and cost saving in the prediction of deficient part in powder blend as compared to conventional trial and error approaches or software-based prediction models. ${ }^{6}$

\section{MATERIAL}

Ramipril Hydrochloride was obtained as a gift sample from Lupine pharmaceutical Industries, Aurangabad, India. Losartan Potassium was purchased from Aristo Laboratories, Mumbai, India. Hydroxy propyl methyl cellulose, Carbopol 934p and Polyvinyl Pyrrolidone-K30 were obtained from the Shreya life Sciences as gift samples.

\section{METHODS}

\section{Preparation of Gastro-bilayer floating matrix Tablets}

\section{Preparation of Immediate-release layer}

The immediate-release layer of Losartan potassium was prepared by mixing drug along with the different excipients and SeDeM expert system applied for evaluation of suitability, critical quality attributes and the behavior of the drug material and excipients used for the 
formulation development. All the ingredients were passed through sieve number 40 based on radius value and loaded in double cone blender and blended for $10 \mathrm{~min}$ at 20 RPM and before compression Magnesium stearate was sifted through sieve number 60 and loaded in the blender and blended for $5 \mathrm{~min}$ at $20 \mathrm{RPM}$. The obtained blend was subjected to12 tests of the SeDeM system and the result obtained was calculated for radius values. ${ }^{7}$ Based on radius values SeDeM diagram was plotted and index values were calculated using equations of homogeneity index, Good Compressibility Index, Parameter Profile Index and mean of all parameters was calculated. ${ }^{8}$ The prepared blend was compressed using $8.5 \mathrm{~mm}$ round beveled edge punches and die set having a plain surface on both sides. In-process quality control tests were performed on the compressed tablets such as disintegration time, thickness, hardness and weight variation.

\section{Preparation of Gastro-floating layer}

The trail floating layer has also been formed with the help of SeDeM expert system. A floating layer formulation utilizes a mixture of sodium bicarbonate and citric acid along with different grades of Hydroxy propyl methyl cellulose K4M, K15M and K100M. Based on formulation trials optimized floating layer was prepared for the sustained delivery of Ramipril with polymers Hydroxy propyl methyl cellulose-K4M, Sodium carboxy methyl cellulose and Carbopol with sodium bicarbonate and Citric Acid as an effervescent mixture. ${ }^{9}$ Floating matrix tablets were prepared by direct compression with $12 \mathrm{~mm}$ flat punches. The prepared tablets were tested for hardness, thickness, weight variation and floating lag time. ${ }^{10}$ The hardness of floating layers was set in the range of $3-5 \mathrm{~kg} /$ $\mathrm{cm}^{2}$ for the total tablet weight of $300 \mathrm{mg} .{ }^{11}$

\section{Preparation of gastro-bilayer Tablets}

SeDeM index values were calculated to convert all the parameter values of an optimized batch to a fixed numerical value for the selection of the Gastro-bilayer formulation process. Different index values were calculated like Parameter index, the Parameter profile index and the Good compression index from these index values, the conclusion can be drawn concerning the compression blend showing the parameter index value $\geq 0.5$ considered as best in terms of compression properties. ${ }^{12}$ Hence Gastro-bilayer tablets were prepared by combining batch IR3 of the immediate-release layer with the floating layer. IR3 batch showed a minimum disintegration time of $90 \mathrm{sec}$ and hence selected for further studies. The $12 \mathrm{~mm}$ diameter punches were used for compression of powder blend with the optimum force for the floating layer; and the required quantity of the immediate release layer IR3 batch was placed over the compressed floating layer and again compressed in the same manner.

\section{In vitro drug dissolution study}

In-vitro dissolution technique is one of the best quality control tools to assess batch to batch release performance of the drug, formulation development and to monitor the manufacturing process. ${ }^{13}$ Drug dissolution test was performed by using USP type II apparatus with 900 $\mathrm{mL}$ of water as the dissolution medium at $50 \mathrm{RPM}$ and $37^{\circ} \mathrm{C}$.

\section{Analysis of Ramipril Hydrochloride and losartan potassium}

Aliquots of $5 \mathrm{ml}$ sample were withdrawn filtered and analyzed for quantitative determination of Ramipril Hydrochloride by UV spectroscopy after each hour for $12 \mathrm{hr} .^{14}$ The quantitative determination of Losartan Potassium was performed by HPLC. The mobile phase was prepared by mixing Acetonitrile, Methanol and phosphoric acid in the ratio of 25:35:20 respectively. The filtered mobile phase components were pumped and the eluent was detected by a UV detector at $\lambda_{\max } 210 \mathrm{~nm} .^{15}$

\section{Fourier Transform Infrared Study}

FTIR Spectra of samples were obtained by mixing about 2-3mg of samples with dried potassium bromide of equal weight and compressed to form a $\mathrm{KBr}$ disc and finally, the sample was scanned from 400 to 4000 $\mathrm{cm}^{-1}$ wave number for all of the formulations giving interactions between the drug and various polymers.

\section{$3^{2}$ factorial design and multiple regression analysis}

$3^{2}$ factorial design was used to find out the best polymer for floating layer and for that two independent factors i.e. HPMC K15M (X1) and Sodium carboxy methyl Cellulose (X2) were selected at 3 levels such as for HPMC K15M (X1) upper 100mg, middle 90mg and lower $80 \mathrm{mg}$, where as for Sodium carboxy methyl Cellulose (X2) upper 60mg, middle $50 \mathrm{mg}$ and lower $40 \mathrm{mg}$. Based on these values 9 factorial batches were prepared. A backward regression analysis technique that was used to generate the best fit models for the analyzed responses and final equation contains only the significant factor terms corresponding to the response obtained from coded and actual values of factors. ${ }^{16}$

\section{RESULTS}

This system provides a physical profile of powder material intended to be used and suggests their flow performance as shown in Figure 1 the compositions of the ingredients are given in Table 1.

Radius values SeDeM diagram was plotted and index values are calculated and mean of all parameters are calculated and shown in Table 2. Various compositions of formulations for Gastro-bilayer tablets and parameters such as hardness, thickness, weight variation and floating lag time are summarized in Table 3 . The pre-compression parameters

Table 1: Formulation parameters of the immediate-release layer.

\begin{tabular}{cccccccccc}
\hline CONTENTS & IR & IR & IR & IR & IR & IR & IR & IR & IR \\
\hline Losartan potassium & 25 & 25 & 25 & 25 & 25 & 25 & 25 & 25 & 25 \\
SSG & 4 & 8 & 12 & - & - & - & - & - & - \\
Crosspovidone & - & - & - & 4 & 8 & 12 & - & - & - \\
Pregelatinized starch & - & - & - & - & - & - & 4 & 8 & 12 \\
PVP-K 30 & 5 & 5 & 5 & 5 & 5 & 5 & 5 & 5 & 5 \\
Dicalcium phosphate & 50 & 46 & 42 & 50 & 46 & 19 & 50 & 46 & 42 \\
Magnesium stearate & 10 & 10 & 10 & 10 & 10 & 10 & 10 & 10 & 10 \\
Talc & 6 & 6 & 6 & 6 & 6 & 6 & 6 & 6 & 6 \\
Total & 100 & 100 & 100 & 100 & 100 & 100 & 100 & 100 & 100 \\
\hline
\end{tabular}


Table 2: Composition of the Gastro-bilayer Tablet.

\begin{tabular}{cccccccccc}
\hline CONTENTS & F1 & F2 & F3 & F4 & F5 & F6 & F7 & F8 & F9 \\
\hline IR3 & 100 & 100 & 100 & 100 & 100 & 100 & 100 & 100 & 100 \\
Ramipril HCL & 2.5 & 2.5 & 2.5 & 2.5 & 2.5 & 2.5 & 2.5 & 2.5 & 2.5 \\
HPMC K 15M & 100 & 100 & 100 & 80 & 80 & 80 & 90 & 90 & 90 \\
NaCMC & 60 & 40 & 50 & 60 & 40 & 50 & 60 & 40 & 50 \\
NaHCO3 & 45 & 45 & 45 & 45 & 45 & 45 & 45 & 45 & 45 \\
Citric Acid & 15 & 15 & 15 & 15 & 15 & 15 & 15 & 15 & 15 \\
Carbopol 934 & 20 & 20 & 20 & 20 & 20 & 20 & 20 & 20 & 20 \\
Mg stearate & 10 & 10 & 10 & 10 & 10 & 10 & 10 & 10 & 10 \\
Talc & 2.5 & 2.5 & 2.5 & 2.5 & 2.5 & 2.5 & 2.5 & 2.5 & 2.5 \\
Lactose & 25 & 15 & 5 & 25 & 15 & 5 & 5 & 5 & 5 \\
Total(mg) & 300 & 300 & 300 & 300 & 300 & 300 & 300 & 300 & 300 \\
\hline
\end{tabular}

IR3-immediate-release batch, HPMC K4M- Hydroxy propyl methylcellulose, Na-CMC: Sodium Carboxy methylcellulose, $\mathrm{NaHCO}_{3}$-sodium bicarbonate

Table 3: SeDeM Index Values.

\begin{tabular}{|c|c|c|c|c|c|c|c|c|c|c|c|}
\hline \multirow[t]{2}{*}{ SeDeM Index } & \multirow[t]{2}{*}{ Limit } & \multirow[t]{2}{*}{ Ramipril } & \multicolumn{9}{|c|}{ Experimental Batch } \\
\hline & & & F1 & F2 & F3 & F4 & F5 & F6 & F7 & F8 & F9 \\
\hline $\begin{array}{l}\text { Parameter } \\
\text { Index }\end{array}$ & $\geq 0.5$ & 0.3 & 0.5 & 0.7 & 0.6 & 0.7 & 0.8 & 0.3 & 0.4 & 0.5 & 0.4 \\
\hline $\begin{array}{l}\text { Parameter } \\
\text { Profile Index }\end{array}$ & $\geq 5.0$ & 3.4 & 4.5 & 6.02 & 5.25 & 5.84 & 5.92 & 3.9 & 4.7 & 5.7 & 4.9 \\
\hline $\begin{array}{l}\text { Good } \\
\text { Compression } \\
\text { Index }\end{array}$ & $\geq 5.0$ & 3.18 & 4.2 & 5.63 & 4.91 & 5.46 & 5.53 & 5.26 & 4.23 & 6.23 & 5.17 \\
\hline $\begin{array}{l}\text { Combined } \\
\text { Value }\end{array}$ & - & 6.88 & 9.21 & 12.36 & 10.75 & 12.0 & 12.24 & 9.16 & 9.33 & 12.43 & 10.47 \\
\hline
\end{tabular}

Table 4: Post-compressional parameters for bilayer Tablets.

\begin{tabular}{cccccc}
\hline $\begin{array}{c}\text { Batch } \\
\text { code }\end{array}$ & $\begin{array}{c}\text { Thickness } \\
(\mathrm{mm})\end{array}$ & $\begin{array}{c}\text { Hardness } \\
(\mathrm{kg} / \mathrm{cm} 3)\end{array}$ & $\begin{array}{c}\text { Friability } \\
(\%)\end{array}$ & $\begin{array}{c}\text { Weight } \\
\text { variation }(\mathrm{mg})\end{array}$ & $\begin{array}{c}\text { Drug content } \\
(\%)\end{array}$ \\
\hline F1 & $6.12 \pm 0.12$ & $5.57 \pm 0.12$ & $0.44 \pm 0.06$ & $300.47 \pm 0.81$ & $99.21 \pm 0.06$ \\
F2 & $6.05 \pm 0.11$ & $5.42 \pm 0.32$ & $0.46 \pm 0.11$ & $301.07 \pm 0.51$ & $99.38 \pm 0.11$ \\
F3 & $6.11 \pm 0.15$ & $5.35 \pm 0.42$ & $0.45 \pm 0.08$ & $302.87 \pm 0.75$ & $100.21 \pm 0.16$ \\
F4 & $6.10 \pm 0.14$ & $5.75 \pm 0.22$ & $0.41 \pm 0.11$ & $301.48 \pm 0.56$ & $99.56 \pm 0.06$ \\
F5 & $6.11 \pm 0.12$ & $5.77 \pm 0.15$ & $0.20 \pm 0.19$ & $300.47 \pm 0.56$ & $100.11 \pm 0.12$ \\
F6 & $6.06 \pm 0.13$ & $5.80 \pm 0.85$ & $0.45 \pm 0.06$ & $300.77 \pm 0.45$ & $98.51 \pm 0.08$ \\
F7 & $6.08 \pm 0.14$ & $5.56 \pm 0.52$ & $0.42 \pm 0.11$ & $301.07 \pm 0.55$ & $100.21 \pm 0.06$ \\
F8 & $6.07 \pm 0.16$ & $5.87 \pm 0.42$ & $0.42 \pm 0.08$ & $300.57 \pm 0.71$ & $100.53 \pm 0.08$ \\
F9 & $6.11+0.17$ & $5.88+0.52$ & $0.41 \pm 0.06$ & $301.47+0.81$ & $100.11 \pm 0.06$ \\
\hline
\end{tabular}

F: formulation code

6\% Sodium starch glycolate containing batch $\left(\mathrm{IR}_{3}\right)$ was selected as it shows the fastest disintegration when compared to other immediate-release formulation.

were evaluated for the Immediate-release layer and the floating layer as per IP specifications through the SeDeM System given in Table 3 and all parameters are within limits of good flowability for all the formulations. In the evaluation of post compressional parameters for prepared tablets such as hardness friability, thickness, weight variation and drug content are summarized in Table 4.
The thicknesses of all tablet formulations are in the range from $6.06 \pm 0.13$ to $6.12 \pm 0.12 \mathrm{~mm}$. The weight of all tablets was uniform between $300.47 \pm 0.81$ to $302.87 \pm 0.75 \mathrm{mg}$. The Hardness of the prepared tablets was found to be between $5.35 \pm 0.42$ to $5.88 \pm 0.52 \mathrm{~kg} / \mathrm{cm}^{2}$. Among all the tablet formulation, F9 showed highest hardness, in the formulation. The hardness data demonstrated that all tablets passed the test as per USP. The friability of the prepared tablets ranged from $0.20 \pm 0.19$ to 


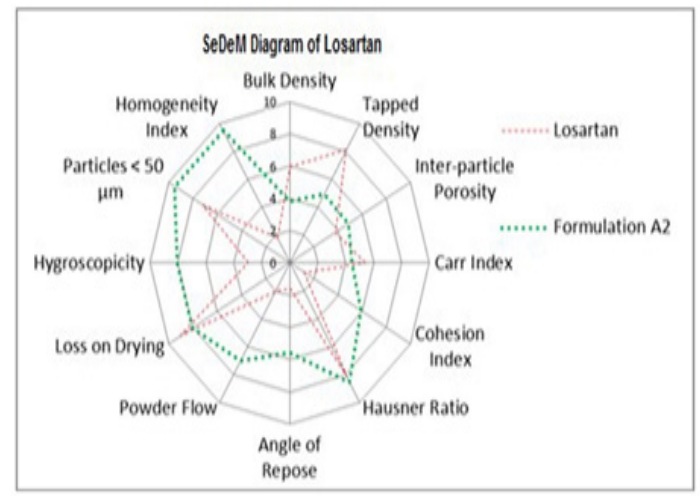

Figure 1: SeDeM Diagram of the immediate-release layer.

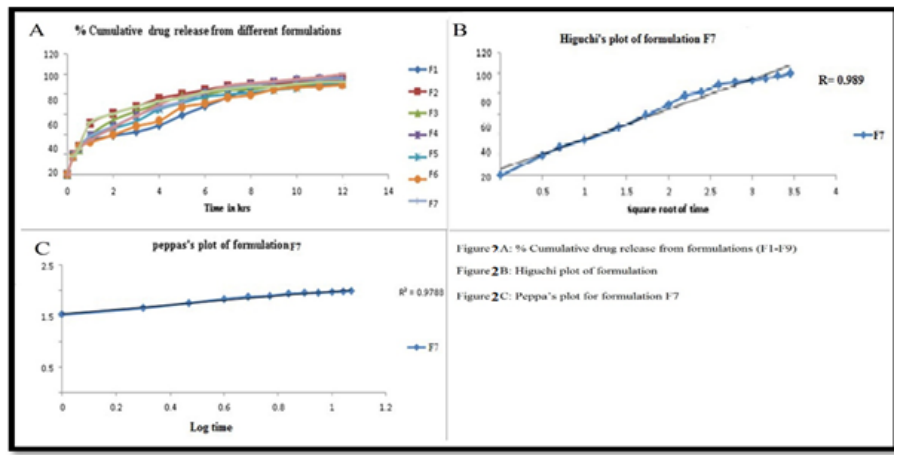

Figure 2: A-Percent Cumulative drug release from formulations (F1-F9), B-Higuchi plot of formulation and C-Peppa's plot for optimized formulation F7.

$0.04 \pm 0.06$. Thus, all tablets passed friability test as per USP as the data was less than $1 \%$. Drug content of all tablet formulations lied within the specified limit, ranged between $99.21 \pm 0.06$ to $100.53 \pm 0.08 \%$. All tablet formulations exhibited good drug content. The drug dissolution profile of F7 was fitted to various kinetic models and the best fit kinetic models for drug release of optimized batch F7 are given in Figure 2. FTIR Interpretation for Losartan Potassium and Ramipril $\mathrm{HCl}$ shows characteristic peaks of $2954.95 \mathrm{~cm}^{-1}$ for C-H stretch, $1577.77 \mathrm{~cm}^{-1}$ for $-\mathrm{NH}, 1259.55 \mathrm{~cm}^{-1}$ for C-N $765.74 \mathrm{~cm}^{-1}$ for C-Cl, $1460.11 \mathrm{~cm}^{-1}$ for $\mathrm{CH}_{2}$ bend and $3650 \mathrm{~cm}^{-1}$ for $\mathrm{OH}$. The swelling and erosion of polymers can be studied by porosity studies which are carried out by Scanning Electron Microscopy and the results are shown in Figure 3.

The cumulative percent of drug released from the formulations was plotted against the time on a log scale and analyzed and Least-Squares Method was referred for linearity studies. Calculating correlation coefficients between time and therefore the \% cumulative drug released on the log-log scale tested the fitness as shown in Figure 4.

\section{DISCUSSION}

From all the factorial Batches F7 is selected as the best formulation for the gastric layer having a combination of Hydroxy propyl methyl cellulose $\mathrm{K} 15 \mathrm{M}$ and Na-CMC which gave 99.57\% drug dissolution within $12 \mathrm{hr}$. From all the formulations based on the dissolution studies batch F7 was selected as the best formulation for the gastric layer. Better sustainable effect and also $99.57 \%$ drug release within $12 \mathrm{hr}$ was obtained in the F7 batch compared to the other formulation.

The swelling and erosion of polymers can be studied by porosity studies, which are carried out by Scanning Electron Microscopy. The tablets

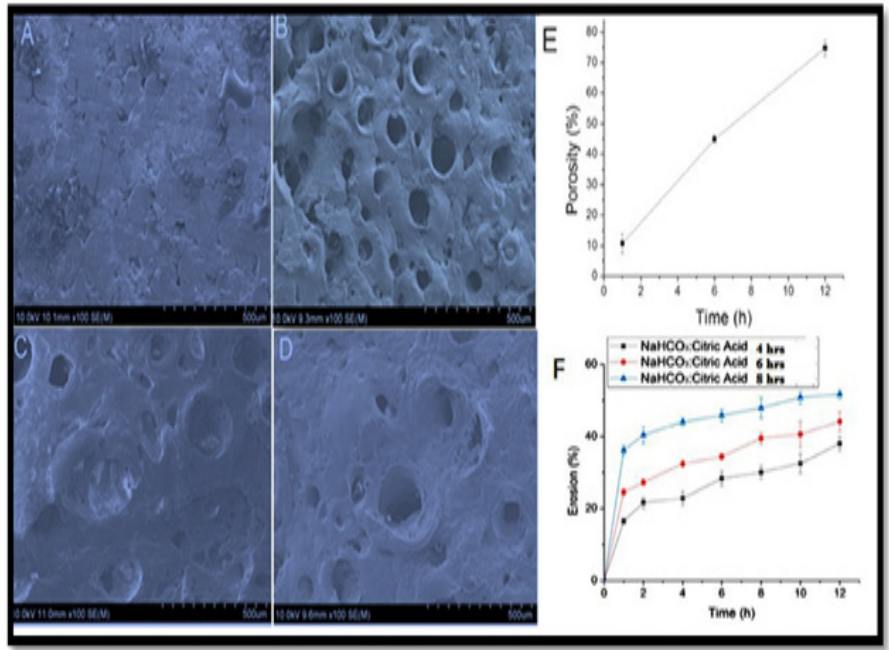

Figure 3: Porosity studies of floating Tablets by scanning electron micrography for batch F7. The Tablets (A) before and after immersion in dissolution medium at (B) $1 \mathrm{hr}$. micrography (C) $6 \mathrm{hr}$ micrography (D) $12 \mathrm{hr}$. micrograph. (E) Percentage of open porosity of Tablets with time. 3(F) the erosion of the tablet after $8 \mathrm{hr}$.

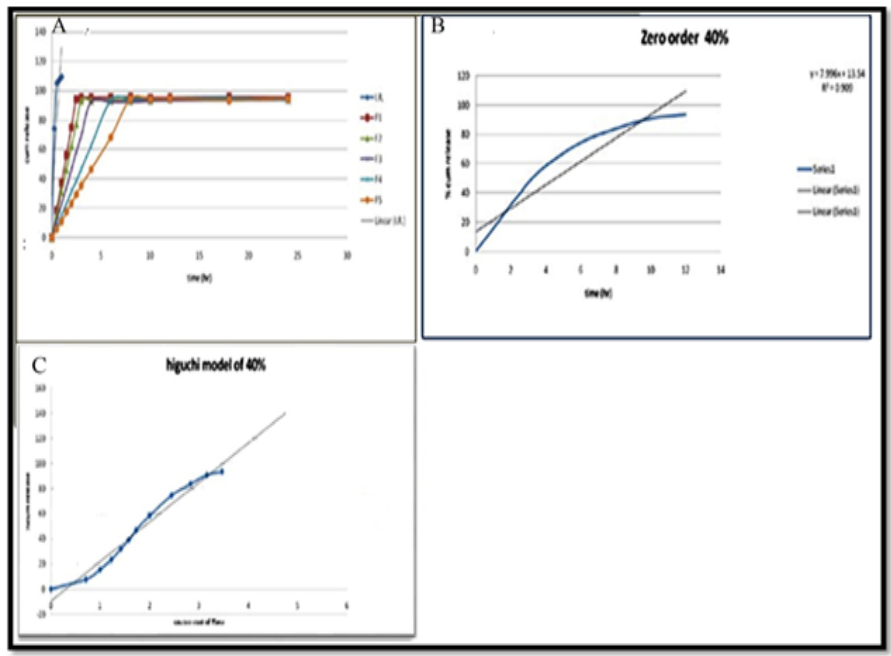

Figure 4: A- In-vitro drug release of the immediate-release formulation. B-Zero-order plot for optimizing Ramipril formulation (F-7). C-Higuchi chart of optimized Losartan formulation (F-7).

exhibited a fast-swelling rate in the initial $6 \mathrm{hr}$. Erosion is mainly responsible for the swelling to be started initially and decreases after 6 hr. Since Hydroxy Propyl methyl Cellulose hydrates the tablet surface the tablet produces a gel layer and slows down the dissolution of Ramipril by preventing the escape of carbon dioxide. Additionally, drug release was calculated by using the Korsmeyer-Peppa's equations and the drug release of the optimized Ramipril hydrochloride was evaluated by standard equations. Korsmeyer-Peppa's model helps in calculating a non-Fickian release type of transport and that the drug release was through both mechanisms i.e. diffusion and erosion but for Ramipril hydrochloride was mainly controlled by erosion.

The drug release however increased with increasing ratio of gasforming agent to Hydroxy Propyl methyl Cellulose. Tablets after its introduction to the dissolution medium can be disintegrated to a larger extent in the initial time because of erosion. Cumulative drug release 
for approximately $12 \mathrm{hr}$ a large amount of drug was released from the tablet, leading to a faster release rate after $4 \mathrm{~h}$ because of presence of polyvinyl pyrrolidone and after $8 \mathrm{hrs}$ the erosion of the tablet was more than $50 \%$. At the same time, the maximum amount of drug has been released from a tablet, so to prevent it the medium has just to wet and penetrate the tablet and the as tablet undergoes erosion as well as swelling because of produce carbon dioxide, the buoyancy of the tablet started to rise onto the medium surface. The amount of gas generating agents did not significantly affect floating time although higher carbon dioxide generation was expected to occur.

In vitro evaluations of gastro-bilayer tablets for Floating lag time and total floating time are important parameters. The main advantage of Shorter Floating lag time is that it prevents early removal of tablets from the stomach and prolonged floating time is essential for enhancing gastric retention time. The present in vitro buoyancy testing in all formulations showed good floating behavior with total floating time greater than $12 \mathrm{hr}$ and floating lag time less than 1 min i.e. between 41 and $55 \mathrm{sec}$. Initial floating was facilitated by gas generation by sodium bicarbonate and citric acid. The formations of pores in the matrix and swelling of tablets is responsible for the reduction in density which helped the tablets to keep floating for a longer period of time. Confirmation of the floating mechanism was through porosity studies by Scanning electron microscopy analysis for optimized batch F7 in which pores were observed at different time interval during dissolution. Pores were formed due to gas generation by sodium bicarbonate and citric acid. With progress in time, pore size and porosity both were increased and gas bubbles entrapped in the gel matrix were also observed at $12 \mathrm{hr}$ which also influenced prolonged floating.

Drug release kinetics was consistent with Higuchi's and Peppa's equation which confirms that drug release was diffusion controlled as evidenced by the correlation values of $R^{2}$. The cumulative amount of Ramipril Hydrochloride released from gastric matrix Tablets at different time intervals was fitted to zero-order kinetics using the Least-Squares method indicating constant drug release. The correlation between the time and thus the cumulative amount of drug released was also calculated for the fitness of the data to zero-order kinetics by different values of the coefficient of correlation.

A full factorial design determines the effect of independent variables on dependent variables at different levels. The data obtained from ANOVA demonstrated the significance of the model with a P-value of less than 0.05 . Consequently, three batches were executed under anticipated conditions of independent variables, i.e. F3, F7 and F9 to confirm the prediction of statistical design. The \% drug release of formulation F7, as predicted by the model, was $99.18 \%$ for Losartan and $99.68 \%$ for Ramipril, respectively which is matching with the drug release in-vitro dissolution. ${ }^{17}$

All the batches have shown that the variation in the polymer concentration affects the drug release rate of Losartan Potassium. Developed Gastrobilayer and the marketed immediate release tablets were compared through in vitro drug release characteristics. Dissolution data for all the experiments were highly reproducible and hence only the average values were plotted. The comparative dissolution profile of prepared Losartan potassium layer with marketed immediate release tablets indicated that more than $90 \%$ of the drug was release within $1 \mathrm{hr}$, which complies with the Pharmacopeial spécifications. In all the batches, It was observed that as the polymer concentration increases the drug release rate decreases. ${ }^{18}$ The pre-compression and post-compression parameters of all the prepared batches were within the prescribed limit as the average percentage deviation of tablets of less than $\pm 5 \%$ as per official requirements of Indian Pharmacopeia. All the other test for tablet formulations were within the acceptable range for properties like weight variation, drug content uniformity, hardness and friability. ${ }^{19}$

In the factorial designs, the drug release mechanism was found to be Non-Fickian, controlled by diffusion and swollen matrix. ${ }^{20}$ Higuchi's kinetics of drug release was observed for optimized batch i.e. F7. Which may be used once a day administration in the management of chronic hypertension. ${ }^{21}$

\section{CONCLUSION}

Gastro-Bilayer Floating Tablets of Losartan as Immediate Release layer and Ramipril as sustained-release floating layer are successfully developed, showed good floating with a minimum floating lag time and can also provide the opportunity of higher dose incorporation without affecting bulkiness of tablets and higher bioavailability can be achieved. The problem of poor solubility of drugs can be solved by optimization of blend through SeDeM expert System. Hydroxy propyl methyl cellulose K15M, K4M and Carbopol 934 are helpful in achieving the sustained effect of the drug for $12 \mathrm{hr}$ by Non-Fickian, controlled diffusion and swollen matrix. Peppa's release model is best suited for the final formulation batches based on the drug release mechanisms. On the basis of the two factor study it can be concluded that the release rate of the drug was retarded by a combination of these polymers HPMC K15M and Sodium carboxy methyl Cellulose are helpful in achieving the sustained release of the drug for $12 \mathrm{hr}$ along.

\section{ACKNOWLEDGEMENT}

The authors wish to thank all those who helped us to do this research work.

\section{CONFLICT OF INTEREST}

The authors declare that there are no competing interests.

\section{ABBREVIATIONS}

RPM: Revolution per minute; UV: Ultraviolet; USP: United States Pharmacopoeia; nm: Nanometer; mm: Millimeter; cm: Centimeter; mg: Milligram; Kg: Kilogram; $\lambda$ : Wavelength.

\section{REFERENCES}

1. Shilpa A, Rama RT, Hussain MA. Formulation and in-vitro evaluation of once-daily sustained release matrix tablets of stavudine. J Pharm Sci Innov. 2012;1(6):64-8.

2. Rangapriya $M$, et al. Sustained release of stavudine from different polymer matrices. Int J Pharm. 2011;3(4):3682-92.

3. Saravanakumar $\mathrm{M}$, et al. Extended-release matrix tablets of stavudine: Formulation and in vitro evaluation. Asian J Pharm. 2010;4(3):219-23.

4. Kambham $\vee$, Shanthi A. Formulation and evaluation of Glipizide matrix tablets. J Pharm Biol Sci. 2012;2(5):17-23.

5. Thirumalesh NSB, Venkateswarlu K, Chandrasekhar KB. Formulation and in vitro evaluation of Pregabalin mini-tablets for sustained release. Pharm Lett. 2016;8(2):277-83.

6. Shahi SR, Tadwee I. Se DeM in preformulation of the solid oral dosage form: A Review. AAPSJ. 2017;1(1):11-7.

7. Thirumalesh NSB, Venkateswarlu K, Chandrasekhar KB. Formulation and in-vitro evaluation of orodispersible tablets of olanzapine for the improvement of dissolution rate. J Chem Pharm Res. 2016;8(1):177-81.

8. Thirumalesh NSB, Venkateswarlu K, Chandrasekhar KB. Formulation and evaluation of Oxybutynin chloride extended-release matrix tablets. Indo Am J Pharm Res. 2016;6(1):4179-84.

9. Vijayabhaskar $\mathrm{K}$, et al. Preparation and in-vitro evaluation of ranitidine mucoadhesive microspheres for prolonged gastric retention. $\mathrm{Br} J$ Pharm Res. 2016;10(2):1-12

10. Venkateswarlu K, Chandrasekhar KB. Development and statistical optimization of sustained-release gastro-retentive floating tablets of cephalexin. Marmara Pharm J. 2016;22(2):172-83. 
11. Metkar V, Kumar A, Pankaj P, Deepti P, Sahu S. Formulation development and evaluation of Bilayer tablets of Lornoxicam. Int J Drug Dev Res. 2012;4(2):173-9.

12. Sawant VA, et al. Plan and in-vitro discharge dynamic investigation of stavudine from maintained discharge network tablet containing hydrophilic and hydrophobic polymers. Indian J Nov Drug Deliv. 2009;1(1):36-41.

13. Bashar A, Alkhalidi A, Alkhatib HS, Khdair AA. Comparative dissolution of diltiazem immediate and extended-release products using conventional and innovative dissolution paddles. Open Drug Deliv J. 2010;4:48-54.

14. Patil SV, Kuchekar BS, Janugade BU, Lade PD. In-vitro studies of SVD sustained release from hydrophilic matrices. J Pharm Res. 2009;2(12):1855-6.

15. Braja BP, Debasis M, Goutam G, Sudhir KP, Puspita A. Evaluation of binder's efficiency of different natural gums in the tableting process. Pharm Lett. 2010;2(4):429-31.

16. Govedarica B, Injac R, Dreu R, Srcic RS. Formulation and evaluation of immediate-release tablets with different types of paracetamol powders prepared by direct compression. Afr J Pharm Pharmacol. 2011;5(1):31-41.

17. Roychowdhury S, Singh H, Deora G, Sharma S. Formulation, evaluation and optimization of enteric-coated tablets of erythromycin stearate by multivariate ANOVA method. AJADD. 2014;2:308-19.

18. Murtada AO, Abdelkarim MA, Huyam AM. The effect of sodium starch glycolate concentration on the physical effects of chlorpheniramine tablets. J Pharm Edu Res. 2013;4(1):47-53.

19. Lifang Y. Gastro-floating tablets of cephalexin: preparation and in vitro/in vivo evaluation. Int J Pharm. 2013;452(1-2):241-8.

20. Rajamma AJ, Yogesha HN, Sateesha SB. Natural gums as sustained-release carriers: development of gastro-retentive drug delivery system of ziprasidone HCl. DARU J Pharm Sci. 2012;20(58):2008-231.

21. Saisivam S, Rahamath UM, Shakeel F. Development of floating matrix tablets of losartan potassium: In vitro and in vivo evaluation. J Drug Del Sci Tech. 2013;23(6):611-7.

Article History: Submission Date : 25-03-2020; Revised Date : 26-06-2020; Acceptance Date : 06-07-2020.

Cite this article: Ahmed SI, Zaheer Z, Khan FN, Hasan M. Formulation and Evaluation of Gastro-Bilayer Floating Tablets of Losartan Potassium as Immediate Release Layer and Ramipril Hydrochloride as Sustained Release Floating Layer. Int. J. Pharm. Investigation, 2020;10(3):294-9 